\title{
Robot Control Using Intelligent Gain Sliding Mode
}

\author{
Azita Yazdanpanah $^{1}$, Dr. Abbas ali Rezaee ${ }^{2}$ and Dr. Ahmad Faraahi ${ }^{2}$ \\ ${ }^{1}$ Engineering faculty of Bushehr's Payam-e-Nour University(asalooyeh) \\ ${ }^{2}$ Department of Computer Engineering, Payame Noor University, 19395-3697, \\ Tehran, Iran \\ yazdanpanah_a8687@yahoo.com
}

\begin{abstract}
In this research, intelligent sliding mode controls are presented as robust controls for robot manipulators. The objective of the study is to design controls for robot manipulators without the knowledge of the boundary of the uncertainties by using an intelligent sliding mode control (SMC) while elucidating the robustness of the fuzzy SMC. A sliding mode control provides for unlimited accuracy in presence of bounded disturbance, although the sliding mode controller also causes chattering. Chattering is undesirable for use with actual component, since it might causes damage to them with a subsequent loss of accuracy. Such chatter is caused by overestimation of the controller gain. An intelligent sliding mode is proposed as a solution to the problems created by chattering; to illustrate, a continuum robot manipulator is simulated with an intelligent sliding mode control. The performance of intelligent gain sliding mode controller is demonstrated through the simulation results. The results of the simulations show the effectiveness for chattering mitigation by means of avoiding overestimation, and the robustness of an intelligent sliding mode control.
\end{abstract}

Keywords: sliding mode controller, intelligent control, fuzzy logic, intelligent sliding gain, robot manipulator, robustness

\section{Introduction}

Designing an effective and robust control for a nonlinear system with unmodeled dynamics and system disturbances/uncertainties is one of the most significant issues facing control engineers [1]. In the real world, system dynamics are seldom fully known, and more unknown disturbances will arise during the operation. Because there are some uncertainties or unknown disturbances within the system, many engineers have developed a dedicated design control which is insensitive to changes in system dynamics; this dedicated control has become known as a robust control [2-3]. The sliding mode control is an example of a dedicated design control that is used as a robust control [4]. The main feature of the sliding mode control is that it is insensitive to disturbances and uncertainties, if those are bounded.

However, the sliding mode control introduces chattering, which is one of the most significant problems in the field of sliding mode control [4-5, 10-14]. Overestimating the boundaries of uncertainties and disturbances leads to high sliding mode controller gains and thus increases chattering. Chattering in systems with a sliding mode control is usually caused by the unmodeled dynamics and can be observed as high frequency (but less than infinity) control switching. The chattering effect in control results in oscillations in the sliding variable dynamics. These oscillations prevent sliding variables from being constrained to zero, which results in the degradation of the accuracy of sliding variable stabilization. As a solution to this issue, a sliding mode control with gain adaptation has been proposed, because of its ability to reduce the chattering; it retains the main properties of a sliding mode control but can also control a system with matched bounded disturbances, in which the bounds are unknown. 
This paper examines and compares two strategies for creating robust controls in an atmosphere of dynamic disturbances and uncertainties. The first strategy utilizes a sliding mode control while the second utilizes a sliding mode control with an intelligent gain control. Using the nonlinear system to compare these strategies, this paper shows that the sliding mode control with gain adaptation is a better strategy to design successful control models for use in the presence of the unknown bounded disturbances or uncertainties with unknown boundaries, since the adaptive gain sliding mode control mitigates chattering.

The robot manipulator is chosen as the nonlinear system. Robot manipulators are designed to move material, parts, tools, and specialized devices by having various programmed motions for different tasks. These manipulators consist of links connected by joints; those connected links then form a kinematic chain. The robot manipulators have several features that make them attractive in an industrial environment. Among the advantages often mentioned are decreased labor costs, increased precision and more humane working conditions, as dull, repetitive, or hazardous jobs are performed by the robot manipulators rather than by humans. The robot manipulators are doing adhesive and welding operations to connect the side frame with the body shell. Typical applications of robot manipulators include welding, painting, assembly, pick and place, and so on. More than 800,000 robot manipulators are in operation in the world, mostly in Japan, the European Union and North America [6-7].

In this work, robust control is designed for the robot manipulators in the presence of unknown disturbances and uncertainties. The joint variables of each robot manipulator's joint are controlled in order to control the motion. As a case study, a continuum robot manipulator is studied and simulated. This paper demonstrates the robustness of the sliding mode control with intelligent gain adaptation through the case study. The results reveal that the sliding mode control with intelligent gain adaptation is an effective method to design a robust control for those multipurpose robot manipulators which perform various tasks.

The structure of the paper is as follows:

Section 2 introduces the robot manipulator dynamics. Section 3 reviews concepts, design techniques of sliding mode control, fuzzy logic theory and paper methodology. Section 4 describes the summary of the results of this paper and in the last part draws a conclusion.

\section{Theory}

\section{Robot Dynamics}

This part introduces the dynamics of robot manipulators. Since its inception, the field of robot dynamics has presented many issues in refining both theory and operations; one of the most challenging areas of study has been the problem of computational efficiency in the dynamics of mechanisms. Many efficient algorithms in dynamics have been developed to address this problem. In this part, using the developed robot dynamics, the inverse dynamics of robot manipulators are derived and used to develop a control design.

The dynamics of robot manipulators illustrate the relationship between force and motion. The generalized force for a robot manipulator can be described as a second-order nonlinear differential equation. The dynamic equation of robot manipulators is derived using the Lagrangian [8]. The Lagrangian is derived by subtracting potential energy from kinetic energy [9]. The following Lagrangian formulation is defined as:

$L=K-P$

where $K$ is defined as the sum of the kinetic energy for each joint, and $P$ is defined as the sum of the potential energy for each joint. Using Lagrangian $L$, Euler-Lagrange Equation is defined as [1] 
$\frac{d}{d t} \cdot \frac{\partial L}{\partial \dot{q}}-\frac{\partial L}{\partial q}=\tau_{k}$

Where $\dot{\boldsymbol{q}}$ is defined as a joint velocity, $q$ is defined as a joint variable, $\boldsymbol{\tau}$ is defined as a generalized force. The equation, which is rewritten Equation (2) in matrix form, is the dynamic equation of robot manipulator.

The model resulting from the application of Lagrange's equations of motion obtained for this system can be represented in the form

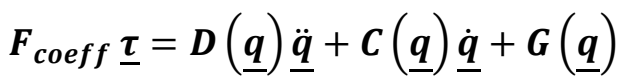

where $\tau$ is a vector of input forces and $\mathrm{q}$ is a vector of generalized co-ordinates. The force coefficient matrix $F_{\text {coeff }}$ transforms the input forces to the generalized forces and torques in the system. The inertia matrix, $D$ is composed of four block matrices. The block matrices that correspond to pure linear accelerations and pure angular accelerations in the system (on the top left and on the bottom right) are symmetric. The matrix $C$ contains coefficients of the first order derivatives of the generalized co-ordinates. Since the system is nonlinear, many elements of $C$ contain first order derivatives of the generalized coordinates. The remaining terms in the dynamic equations resulting from gravitational potential energies and spring energies are collected in the matrix $G$.

\section{Methodology}

The main advantage of sliding mode control is the robustness that can be achieved when properly matched to the bounded disturbances. This part begins by introducing the concepts of the sliding surface and the sliding mode. Then, it demonstrates how to design 1) a sliding mode control and 2) an intelligent gain adaptive sliding mode control.

All the values in the robot manipulator dynamic equation are known or measurable, except for the boundary of the bounded disturbances and uncertainties and joint velocities. The robot manipulator is controlled via joint variables, and the commands are given as joint variables.

The goal of the intelligent gain sliding mode control for the robot manipulator is to drive the joint variables to the desired or command values in finite time and keep them thereafter in the presence of the bounded disturbances, without overestimation of controller gains. The following is the design procedure for the control system for the robot:

- Estimate joint velocities using a super-twisting observer.

- Design sliding variables of the robot manipulator control system for each control.

- Derive control $u$.

- Design a gain adaptation for each control.

It is now necessary to design a sliding functions for the traditional sliding mode control of the robot manipulator as follows [10-14],

$V=\frac{1}{2} S^{T} . D . S$

Then the derivative of the sliding variables is expressed as,

$\dot{V}=\frac{1}{2} S^{T} \cdot \dot{D} \cdot S+S^{T} D \dot{S}$ 
Therefore, using the Lyapunov function, the following inequality is derived

$D \dot{S}=-V S+D \dot{S}+V S+G-\tau$

Then the row of control function is designed as follows:

$\dot{V}=\frac{1}{2} S^{T} \dot{D} S-S^{T} V S+S^{T}(D \dot{S}+V S+G-\tau)=S^{T}(D \dot{S}+V S+G-\tau)$

suppose the control input is written as follows

$\widehat{\tau}=\widehat{\tau_{e q}}+\widehat{\tau_{d l s}}=\left[\widehat{D^{-1}}(\widehat{V}+\widehat{G})+\dot{S}\right] \widehat{D}+K \cdot \operatorname{sgn}(S)+K_{v} S$

The control derived in Equation (8) is a high frequency switching function which causes chattering. It is necessary to design control using an intelligent sliding variable to eliminate chattering. The intelligent sliding variable is described in the following parts.

$$
\begin{aligned}
& \dot{V}=S^{T}\left(D \dot{S}+V S+G-\widehat{D} \dot{S}-\widehat{V} S-\widehat{G}-K_{v} S-K \operatorname{sgn}(S)=S^{T}(D \dot{S}+\widetilde{V} S+\widetilde{G}-\right. \\
& \left.K_{v} S-K \operatorname{sgn}(S)\right)
\end{aligned}
$$

The controller gain $(K)$ is necessary to satisfy the following condition

$$
\left|\widetilde{D} \dot{S}+\widetilde{V} S+\widetilde{G}-K_{v} S\right| \leq K
$$

to provide the finite time convergence.

The Lemma equation in robot manipulator system can be written as follows

$$
K_{u}=\left[|\widetilde{D} \dot{S}|+|V S|+|G|+\left|K_{v} S\right|+\eta\right]_{i}, \quad i=1,2,3,4, \ldots
$$

Due to above formulations, we have:

$$
\dot{V} \leq-\sum_{i=1}^{n} \eta_{i}\left|S_{i}\right|
$$

Therefore the dynamic formulation for SMC is:

$$
\boldsymbol{U}=\boldsymbol{U}_{\text {eq }}+\boldsymbol{U}_{\text {dis }}
$$

$\boldsymbol{U}_{\boldsymbol{e q}}$ can be calculate as follows:

$$
U_{e q}=\left[D^{-1}(f+C+G)+\dot{S}\right] D
$$

$\boldsymbol{U}_{\boldsymbol{d i s}}$ is computed as;

$$
U_{\text {sat }}=K \cdot \operatorname{SGN}(S)
$$

Fuzzy-logic aims to provide an approximate but effective means of describing the behavior of systems that are not easy to describe precisely, and which are complex or ill- 
defined. It is based on the assumption that, in contrast to Boolean logic, a statement can be partially true (or false). For example, the expression (My University is near Shiraz) where the fuzzy value (near) applied to the fuzzy variable (distance), in addition to being imprecise, is subject to interpretation. The essence of fuzzy control is to build a model of human expert who is capable of controlling the plant without thinking in terms of its mathematical model. As opposed to conventional control approaches where the focus is on constructing a controller described by differential equations, in fuzzy control the focus is on gaining an intuitive understanding (heuristic data) of how to best control the process, and then load this data into the control system.

The central idea of fuzzy sets is that elements can have partial membership in a given set. In contrast to a classical set, a fuzzy set, as the name implies, is a set without a crisp boundary. In this respect, fuzzy sets are functions that map a value to a number between zero and one, indicating its actual degree of membership. A degree of zero means that the value is not in the set, and a degree of one means that the value is completely representative of the set. They were introduced by Prof. Lotfi A. Zadeh in 1965 as an extension of the classical notion of "set" and as a mathematical way to represent and deal with vagueness in everyday life. The fuzziness does not come from the randomness of the constituent members of the set, but from the uncertainties and imprecise nature of abstract thoughts and concepts.

A Fuzzy-Logic System (FLS) is a non-linear mapping from the input to the output space, where the input is first fuzzified as shown in Figure 1.

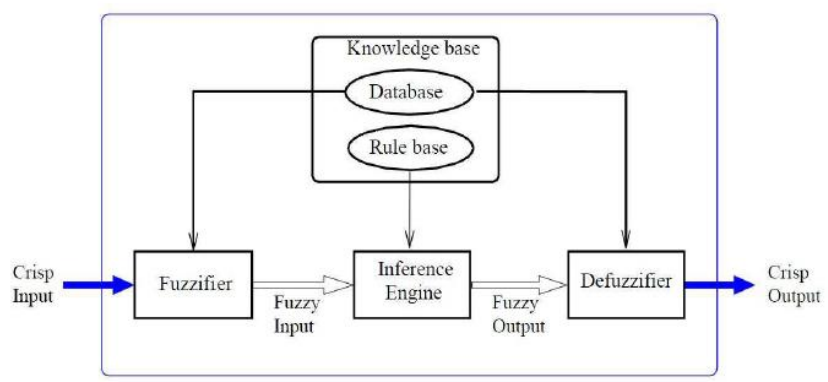

Figure 1. Implementation of Fuzzy-logic Control System, Adapted From

The fuzzy sets computed by the fuzzy inference as the output of each rule are then composed and defuzzified. Fuzzification helps in evaluating the rules, but the final output of a fuzzy system has to always be a crisp number. (i.e., conversion from a fuzzy set to a crisp number). Fuzzy membership functions on the other hand, are defined in terms of numerical values of an underlying crisp attribute. For example: Short, Medium and Tall in terms of the fuzzy variable: height. In other words, determining how much each discrete input value belongs to each input fuzzy set using the corresponding membership function. The next sections will explain the operations of every module in Figure 1 in order to formulate the non-linear parametrised mapping for the fuzzy-flatness tracking control.

Fuzzification is the process of translating crisp input values into fuzzy linguistic values (fuzzy sets) through the use of membership functions. Generally, fuzzy membership functions are defined in terms of numerical values of an underlying crisp attribute such as short, medium and tall in terms of the fuzzy variable 'Height'. They are subsequently processed by the inference engine that retrieves knowledge in the form of fuzzy rules contained in the knowledge-base.

Fuzzy knowledge-bases are implemented as a set of IF-THEN rules as follows:

IF (condition1 AND/OR condition2) THEN (consequence). 
In fuzzy logic terminology, the statement following the IF is known as the premise, antecedent, or condition. The corresponding statement following THEN is known as the conclusion or consequent, and the actual calculation of the consequent using the premises calculated from the fuzzified inputs is reserved for the inference engine [11-14].

In designing fuzzy systems, one should decide whether the number of rules is sufficient and if there are specific interactions between the rules. These problems were discussed in detail in the works.

The inference engine is the heart of a FLC and acts as the bridge between the fuzzification and defuzzification stages. It aims at translating the designers desired control rules from a linguistic representation to a numeric computation, and can be divided into three elements: aggregation, composition, and accumulation. There are several types of FIS, which may be limited to two FIS, the most currently used, those of Takagi-Sugeno or Mamdani type. The performance of any fuzzy logic controller (FLC) is greatly dependent on its inference rules and can be drastically affected by the choice of membership functions. Thus, methods for tuning the fuzzy logic controllers are needed. Some applications considered neural networks and genetic algorithms to solve the problem of tuning a fuzzy-logic controller.

In general, the system to be controlled using a FLC requires a crisp or discrete input, rather than a membership function that is produced by the inference engine. Defuzzification is the process of converting the fuzzy output set resulting from the inference process into a discrete number suitable for input to the plant. There are many different methods of defuzzification described in the literature, with varying levels of complexity. Two fundamental methods are known as the Mean of Maxima (MoM) method and the Mamdani's Center of Gravity ( $\mathrm{CoG})$ method. In the current work, the Mamdani's COG method shall be used as will be discussed in the following.

The synthesis of the fuzzy-logic control in the current work will be limited to the intelligent tracking of the computed torque control's desired trajectories. This will be established by implementing an off-line tuning of the pole placement coefficients at each excitation frequency without recourse to the tedious. Figure 2 shows the fuzzy logic solutions of the computed torque control.

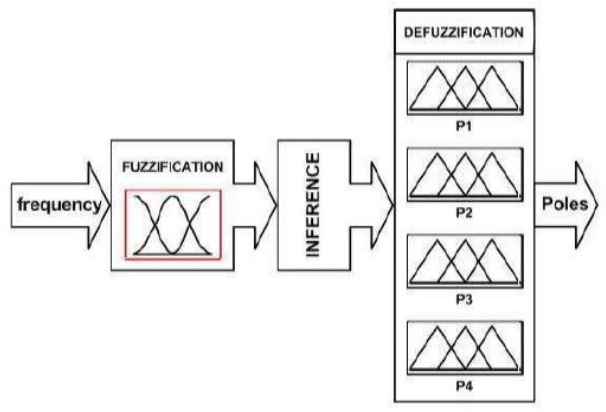

\section{Figure 2. Fuzzy Logic Solutions of the SMC}

This sliding mode control with fuzzy gain adaptation provides discontinuous control function without overestimating the boundary of the disturbances/uncertainties. Chattering can be observed in the output. For reduce the chattering an intelligent gain sliding mode control is defined as follows:

$A=\alpha \cdot A_{T}+(1-\alpha) \cdot(\dot{e}+\lambda e)=\alpha\left(\lambda \cdot e^{P}+\dot{e}\right)+(1-\alpha) \cdot((\dot{e}+\lambda e))$

$\alpha$ is the output of fuzzy logic gain sliding mode controller. Therefore the fuzzy gain sliding mode controller is: 
$\tau=H(q)\left(\alpha \cdot A_{T}+(1-\alpha) \cdot(\dot{e}+\lambda e)\right)+N(q, \dot{q})$

\section{Results and Discussion}

In this section, compare the results of the simulations and evaluate the controllers for robot manipulator.

Tracking data: Figure 3 shows the tracking data in two type's methodology: sliding mode control and intelligent gain sliding mode control. Due to the following graph, in certain conditions sliding mode control has chattering but intelligent gain SMC can remove the chattering.

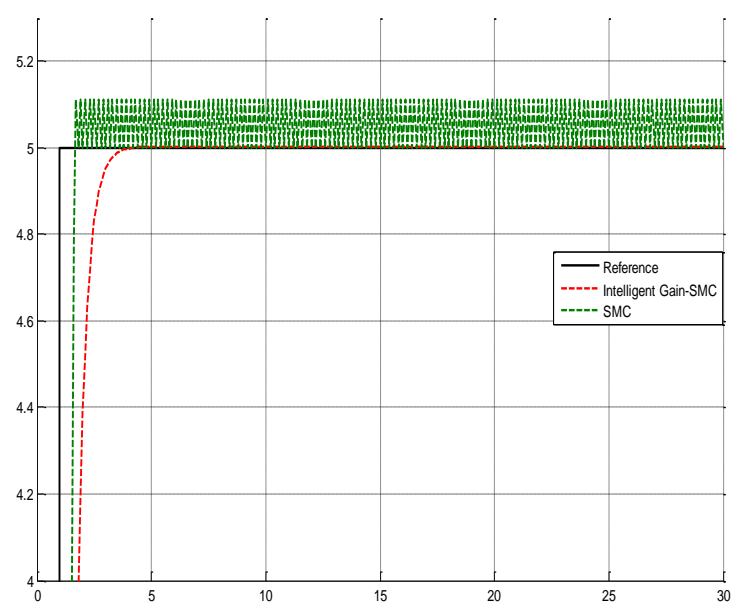

Figure 3. Trajectory Data in SMC and Intelligent Gain SMC

Sliding Variable: Figure 4 illustrates the comparison of sliding variables in SMC and intelligent gain SMC. According to the following graph, the sliding mode controller has chattering but intelligent gain sliding mode controller remove the chattering in sliding variables in presence of unlimited uncertainties.

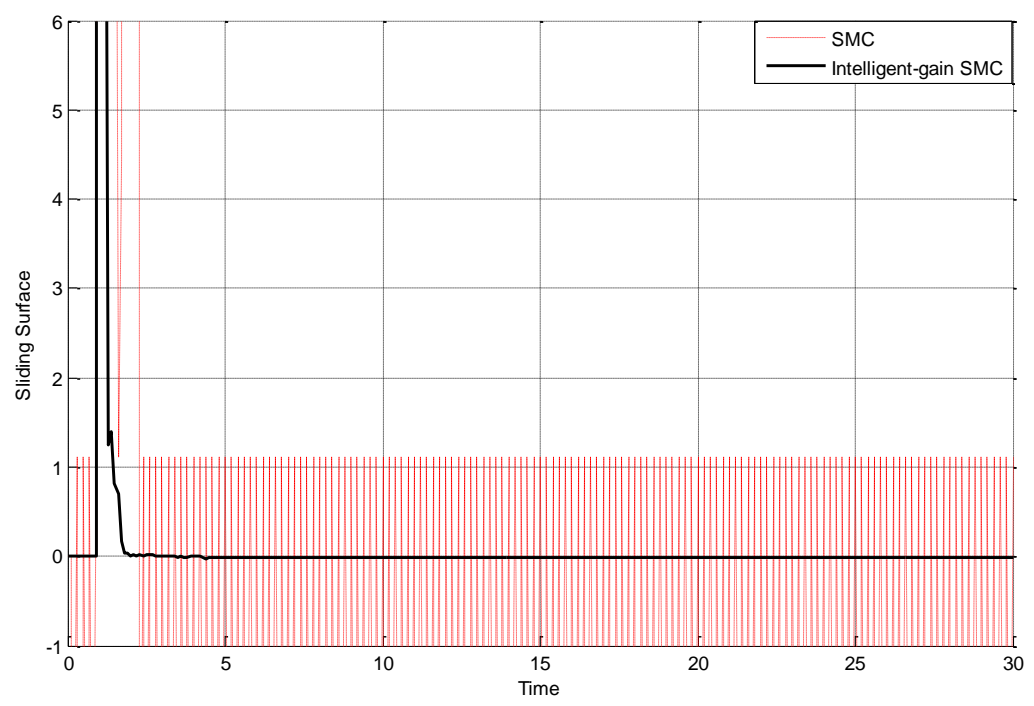

Figure 4. Sliding Variables in SMC and Intelligent Gain SMC 


\section{Conclusion}

This research has studied intelligent gain sliding mode control design for the dynamics of robot manipulators without knowledge of the boundary of the disturbances/uncertainties, using fuzzy sliding mode controls as robust controls for the robot manipulators and as a solution to the problem of chattering. To elucidate this, an intelligent sliding mode control for the n-link robot manipulator which includes the unknown bounded disturbances and uncertainties is studied. A sliding mode control is introduced as a robust control, which provides ultimate accuracy in the presence of the matched bounded disturbances/uncertainties. Chattering is introduced as a problem that accompanies the introduction of a sliding mode control; the chattering may actually damage components and cause loss of accuracy of output. As a solution, gain adaptation is introduced. Gain adaptation mitigates chattering by decreasing the controller gain dynamically when the controller gain is overestimated.

The robot manipulator is chosen as a nonlinear system with un-modeled dynamics, and each adaptive sliding mode control is designed for the robot manipulator system without knowledge of the boundary of the disturbances/uncertainties. As a case study, a robot manipulator is simulated using the designed observers and the designed adaptive controls.

In the case study, traditional sliding mode controllers and intelligent gain sliding mode controllers are designed for the robot manipulator. The control algorithm of the robot manipulator is to control the joint position/velocity to drive the robot manipulator's end effector to a certain position/motion. The boundary of the disturbances/uncertainties and the joint velocity are assumed as unknown. A traditional sliding mode control is applied to design the control of the robot manipulator. Each joint velocity is estimated with the super-twisting observer. The simulation results for each control design are shown. The efficacy of the intelligent gain sliding mode control for the robot manipulators has been confirmed in comparison of the simulation results. The intelligent sliding mode control establishes the sliding mode via the sliding mode control laws with gain adaptation, without knowledge of the boundary of the bounded disturbances/uncertainties. The controller gain values are automatically set up and, because these controller gain values are not overestimated, the chattering is mitigated. The tracking errors, chattering in outputs, and controller gains of the simulations of adaptive-gain sliding mode controls are much close to those of the simulations of traditional sliding mode control with knowledge of the boundary of the disturbances. The greatest achievement of this study is that the intelligent-gain sliding mode control which is designed without knowledge of the boundary of the disturbances, has potential to achieve the accuracy, less chattering, and lower controller gain which are established by the sliding mode control with knowledge of the boundary of the disturbances. To conclude, the intelligent-gain sliding mode control is demonstrated to be the robust control for the unmodeled system, and an effective algorithm to avoid overestimation of controller gains and to mitigate chattering by means of gain adaptation.

\section{References}

[1] M. W. Spong, S. Hutchinson and M. Vidyasagar, "Robot Modeling and Control", John Wiley \& Sons, Inc. (2006).

[2] M. M. Fateh, "Dynamic Modeling of Robot Manipulators in D-H Frames", World Applied Sciences Journal vol. 6, no. 1, (2009), pp. 39-44.

[3] P. Shubhi, "Higher Order Sliding Mode Controller for Robotic Manipulator", 22nd IEEE International Symposium on Intelligent Control Part of IEEE Multi-conference on Systems and Control Singapore, 1-3 (2007) October.

[4] C. Edwards and S. Spurgeon, "Sliding Mode Control", 1st ed. Bristol, PA: CRC Press, (1998).

[5] Y.-J. Huang, T.-C. Kuo and S.-H. Chang, "Adaptive Sliding Mode Control for Nonlinear Systems with Uncertain Parameters", IEEE Transactions on System, Man, and Cybernetics, (2008). 
[6] H. Lee and V. I. Utkin, "Chattering Suppression Methods in Sliding Mode Control Systems", Annual Reviews in Control, (2007).

[7] F. Plestan, Y. Shtessel, V. Bregeault and A. Poznyak, "Adaptive sliding mode control for a class of MIMO nonlinear systems application to an electropneumatic actuator", (2010).

[8] F. Plestan, Y. Shtessel, V. Bregeault and A. Poznyak, "New methodologies for adaptive sliding mode control", vol. 156, (2010).

[9] K. D. Young, V. I. Utkin and U. Ozguner, “A Control Engineer's Guide to Sliding Mode Control”, IEEE TRANSACTIONS OF CONTROL SYSTEMS TECHNOLOGY, vol. 7, no. 3, (1999).

[10] F. Piltan, M. H. Yarmahmoudi, M. Shamsodini, E. Mazlomian and A. Hosainpour, "PUMA-560 Robot Manipulator Position Computed Torque Control Methods Using MATLAB/SIMULINK and Their Integration into Graduate Nonlinear Control and MATLAB Courses", International Journal of Robotics and Automation, vol. 3, no. 3, (2012).

[11] F. Piltan, S. Emamzadeh, Z. Hivand, F. Shahriyari and Mina Mirazaei, "PUMA-560 Robot Manipulator Position Sliding Mode Control Methods Using MATLAB/SIMULINK and Their Integration into Graduate/Undergraduate Nonlinear Control, Robotics and MATLAB Courses", International Journal of Robotics and Automation, vol. 3, no. 3, (2012).

[12] A. Barzegar, F. Piltan, M. Vosoogh, A. M. Mirshekaran and A. Siahbazi, "Design Serial Intelligent Modified Feedback Linearization like Controller with Application to Spherical Motor", Journal of Information Technology and Computer Science, vol. 6, no. 5, (2014), pp.72-83. DOI: 10.5815/ijitcs. 2014.05.10 (DOAJ, DOI: 10.5815).

[13] F. Matin, F. Piltan, H. Cheraghi, N. Sobhani and M. Rahmani, "Design Intelligent PID like Fuzzy Sliding Mode Controller for Spherical Motor", International Journal of Information Engineering and Electronic Business, vol. 6, no. 2, (2014), pp. 53-63. DOI: 10.5815/ijieeb. 2014.02.07(DOAJ, DOI: 10.5815).

[14] M. Rahmani, F. Piltan, F. Matin, H. Cheraghi and N. Sobhani, "Design Intelligent System Compensator to Computed Torque Control of Spherical Motor", International Journal of Intelligent Systems and Applications, vol. 6, no. 8, (2014), pp. 87-96. DOI: 10.5815/ijisa.2014.08.10 (DOAJ, DOI: 10.5815) 
International Journal of Hybrid Information Technology Vol.8, No.1 (2015) 